\title{
Single tensionless transition in the Laplacian roughening model
}

\author{
Juan Jesús Ruiz-Lorenzo, ${ }^{1}$ Esteban Moro, ${ }^{2}$ and Rodolfo Cuerno ${ }^{2}$ \\ ${ }^{1}$ Departamento de Física, Facultad de Ciencias, Universidad de Extremadura, 06071 Badajoz, \\ and Instituto de Biocomputación y Física de los Sistemas Complejos, 50009 Zaragoza, Spain \\ ${ }^{2}$ Departamento de Matemáticas and Grupo Interdisciplinar de Sistemas Complejos (GISC), \\ Universidad Carlos III de Madrid, Avenida de la Universidad 30, E-28911 Leganés, Spain
}

(Dated: February 6, 2008)

\begin{abstract}
We report large scale Monte Carlo simulations of the equilibrium discrete Laplacian roughening (dLr) model, originally introduced as the simplest one accommodating the hexatic phase in two-dimensional melting. The dLr model is also relevant to surface roughening in Molecular Beam Epitaxy (MBE). We find a single phase transition, possibly of the Kosterlitz-Thouless type, between a flat low-temperature phase and a rough, tensionless, high-temperature phase. Thus, earlier conclusions on the order of the phase transition and on the existence of an hexatic phase are seen as due to finite size effects, the phase diagram of the dLr model being similar to that of a continuum analog previously formulated in the context of surface growth by MBE.
\end{abstract}

PACS numbers: 64.60.Cn, 68.35.Ct, 68.35.Rh

Two-dimensional (2D) melting has played a driving rôle in Statistical Physics for more than two decades. Efforts made at clarifying its nature [1] have aided to understand systems in which topological defects are relevant, from the equilibrium fluctuations of metallic surfaces 2 to superfluidity and superconductivity in thin films, and phase transitions in liquid crystals [3]. One of the most intriguing related notions is the hexatic phase, between a solid at low temperature $(T)$ and an isotropic fluid at high $T$, transitions between phases being of the Kosterlitz-Thouless (KT) type. Such is the KosterlitzThouless-Halperin-Nelson-Young (KTHNY) mechanism for 2D melting [3]. Although controversial for some time, the hexatic phase has indeed been found in atomistic model systems [4] and in experiments [5].

A successful approach to systems with defect-mediated phase transitions as the above has been the use of duality to formulate equivalent height models. E.g., the discrete Gaussian (dG) model [Eq. (11) below for bending rigidity parameter $\kappa=0$ ] is dual of the 2D Coulomb gas, and the roughening transition in the former corresponds [6] to the well-known KT phase transition of the latter, driven by the unbinding of vortex-antivortex pairs. With a similar philosophy, the discrete Laplacian roughening (dLr) model was introduced by Nelson [7] to describe 2D melting. Its Hamiltonian is

$$
\mathcal{H}=\frac{1}{2} \sum_{\mathbf{r}}\left(\sigma\left[\nabla_{d} h(\mathbf{r})\right]^{2}+\kappa\left[\nabla_{d}^{2} h(\mathbf{r})\right]^{2}\right)
$$

where $\mathbf{r}$ denotes position on a $2 \mathrm{D}$ lattice of lateral size $L, \nabla_{d}$ is discrete gradient, and $h \in \mathbb{Z}$. The original $\mathrm{dLr}$ model [7] is obtained by setting to zero the surface tension parameter $\sigma$. Note, the $\mathrm{dLr}$ model is a discrete version of the linear approximation to Helfrich's energy functional for 2D membranes [8], and provides a simplified description of fluctuating tensionless surfaces, such as biological membranes [8] or, e.g., such as those grown under conditions typical in Molecular Beam Epitaxy (MBE) [9].
For the dLr model, the KTHNY mechanism would imply [1] an intermediate hexatic phase in which the surface disorders in heights, but not in slopes (quasi-long range orientational order). For low $T$, the surface would be in a flat phase, dual of the isotropic fluid in melting, while for high $T$ the surface would disorder in heights and slopes, providing the dual of the solid phase. In terms of the surface structure factor $S(\mathbf{q})=\langle\hat{h}(\mathbf{q}) \hat{h}(-\mathbf{q})\rangle[10]$, the rough high $T$ phase implies power law behavior as $S(\mathbf{q}) \sim q^{-4}$, changing to $S(\mathbf{q}) \sim q^{-2}$ in the hexatic phase [11], and to existence of a finite correlation length in the flat low $T$ phase. Equivalently, for the stationary height-difference $C(r)$ and slope-difference $C_{d}(r)$ correlations 12], these behaviors amount to: $(i)$ rough phase $C(r) \sim r^{2} \log r$, $C_{d}(r) \sim \log r ;(i i)$ hexatic phase $C(r) \sim \log r, C_{d}(r) \sim 1$; (iii) flat phase $C(r) \sim 1, C_{d}(r) \sim 1$. Results supporting this picture were obtained on small $(L \leq 32)$ square and triangular lattices 13]. However, conflicting evidence for $L \leq 64$ was presented that the model had a single first order transition, see 14] and references therein. The discrepancy has remained unsolved, in spite of recent analytical studies [15], elucidation of the phase diagram being important to the diverse contexts mentioned above.

Here, we provide new Monte Carlo (MC) simulations of the dLr model on the square and triangular lattices. Our results for sizes up to $512 \times 512$, much larger than those previously studied [13, 14], allow us to see previous works as inconclusive due to finite size effects. The model has a single continuous transition, possibly of the KT type, between the flat and the rough phases, there being no sign of an hexatic phase to within our numerical resolution in $T$. Notably, this provides an instance of a roughening transition in which the rough phase corresponds to a free tensionless surface, rather than a free surface with tension, as in the $\mathrm{dG}$ model. Moreover, the phase diagram of the dLr model is seen to resemble closely that of a continuum model proposed [9, 15] for MBE growth, suggesting that both models are in the same universality class, much like the relationship between the $\mathrm{dG}$ and the 

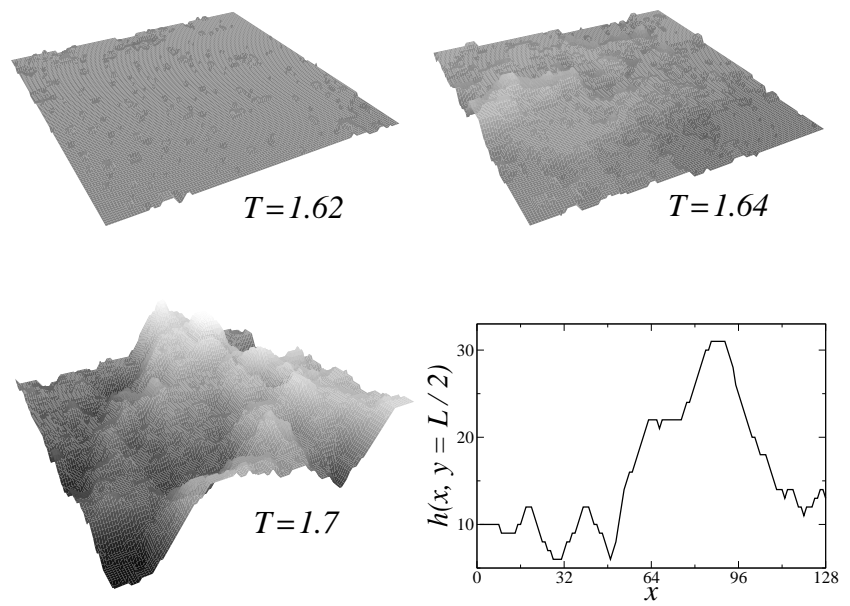

FIG. 1: Surface morphologies for three sample temperatures around $T_{c}$ on the $L=128$ square lattice for Neumann (zero derivative) boundary conditions. Inset: Lateral cut of the surface for $T=1.7$. All units are arbitrary.

continuum sine-Gordon models [16].

For our MC simulations we follow the same procedure as in [13], fluctuations being treated by the histogram method [17], further validated through additional simulations on different points of the extrapolated intervals. Thermalization has been checked by monitoring the behavior of non-local observables like the specific heat and the structure factor at the smallest wave-vector on our finite lattices, $S(q=2 \pi / L)$, as functions of MC time. Note that the dLr model has a richer ground state structure than the dG model, Hamiltonian (11) with $\sigma=0$ being minimized not only by configurations with uniform heights, but also by configurations with uniform slopes (and by more complex morphologies, see below), see e.g. the surface morphology made up of patches with various constant slopes shown in Fig. [- for high $T$. In simulations, this requires large enough system sizes and appropriate boundary conditions so that the full minima structure can be significatively probed. In particular, for small sizes and periodic boundary conditions the system is effectively constrained to fluctuating around a single energy minimum (the morphology with zero slope), inducing apparent hystheretic behavior associated with a first order transition [14]. In our simulations, we have employed both periodic and free (Neumann) boundary conditions, and we have made sure that results provided are (qualitatively) independent of these.

As done for the dG model in [? ], we study the phase transition through the behavior of the structure factor $S(q)$ for different temperatures. In order to test the KTNHY mechanism, we have studied the behavior of $S(q)$ as a function of $T$ and $L$, by fitting the small wavevector part of $S(q)$ to $S(q) \sim q^{-r}$. As seen in Fig. 3 for the square lattice (for the sake of clarity, we omit plots for the triangular lattice, in which completely analogous

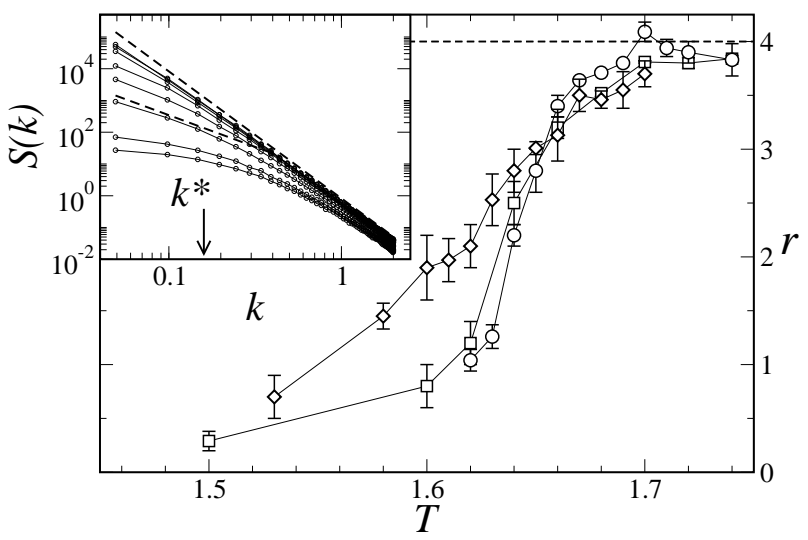

FIG. 2: Effective exponent $r$ in the small wave-vector behavior $S(k) \sim k^{-r}$ (for $\left.k \equiv 2 \sin (q / 2)<k^{*}(L) \approx 3 \pi / L\right)$, as a function of $T$, for $L=32(\diamond), 64(\square)$, and 128 (o). Inset: surface structure factor $S(k)$ on the $L=128$ square lattice vs $k$, for $T=1.62$ (bottom) up to $T=1.69$ (top). Dashed reference lines have slopes -2 (bottom) and -4 (top). All other lines are guides to the eye. All units are arbitrary.

results are obtained), there is no evidence of a finite temperature interval within which $r \simeq 2$, that would be the signature of the hexatic phase. Rather, we find a gradual change from the flat phase behavior $(r \simeq 0)$ to the rough phase one $(r \simeq 4)$. This change becomes more abrupt when the system size is increased, so that only the flat and the rough phases remain well-defined in the thermodynamic limit. These results may thus explain the apparent observation of an hexatic phase in 13. for small $L$ values, where no systematic finite size effects were assessed. By defining the critical temperature $T_{c}$ as the value at which curves for different system sizes cross [17], we estimate $T_{c}=1.65(1)$ for the square lattice and $T_{c}=1.90(2)$ for the triangular lattice.

Further evidence on the existence of a single phase transition is provided by the behavior the specific heat $c(T, L)=\left(\left\langle\mathcal{H}_{\mathrm{dLr}}^{2}\right\rangle-\left\langle\mathcal{H}_{\mathrm{dLr}}\right\rangle^{2}\right) /\left(T^{2} L^{2}\right)$ as a function of temperature. Fig. [1 shows $c(T)$ on the square and triangular lattices for the largest system sizes in our simulations. Within our statistics, a single peak at $T=T^{*}$ can be detected, rather than two as would be expected within the KTHNY scenario. The height and position of the peak are functions of lattice size $L$. Fig. [2 (inset) provides the results of finite size analysis on the specific heat curves, in which the maximum value $c_{\max }(L)$ obtained for each lattice size is plotted as a function of $L$. Remarkably, although for lattice sizes $L \lesssim 70$ the specific heat grows approximately as $c_{\max } \lesssim L$ - compatible with claims on the apparent weakly first order character of the transition for $L \leq 64$ 14] - , for larger $L$ values the increase of $c_{\max }(L)$ slows down. For our largest simulated systems, the best fit is $\operatorname{logarithmic} c_{\max } \sim \log L$. Actually, for the 2D XY model the specific heat at the transition temperature is known 18 to first grow logarithmically with system size and then saturate for large 


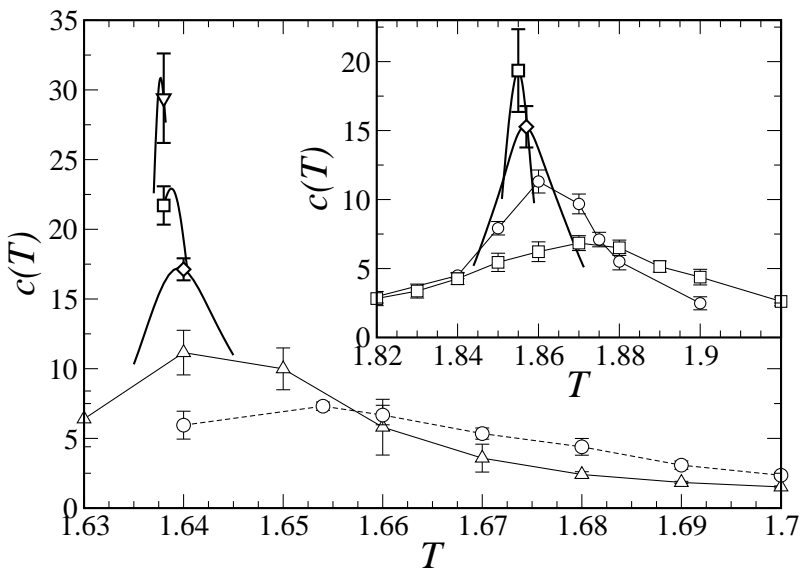

FIG. 3: $c(T)$ vs $T$ on the square (main panel) and triangular (inset) lattices for $L=16(*), 32(\triangle), 64($ o), $128(\diamond), 256$ $(\square)$, and $512(\nabla)$. Bars are statistical errors and thin lines are guides to the eye. For the larger $L$ values in each case, thick solid lines show the $c(T)$ curve extrapolated by the histogram method [17]. All units are arbitrary.

enough values of $L$, suggesting our result might reflect finite size effects. Indeed, saturation is expected provided that the correlation length at $T^{*}$ is smaller than $L$ and thus a horizontal plateau would occur at low $k$ for $S(k)$, namely $r=0$ as defined in Fig. 2] The steady decrease of $r$ at $T^{*}$ for increasing $L$ indicates that such a condition has not being reached. Persistence of logarithmic behavior in the $L \rightarrow \infty$ limit would rather suggest that the phase transition is in e.g. the 2D Ising class [19]. In order to explore this possibility, in Fig. 2 we study the dependence of the specific heat jump position $T^{*}(L)$ with lateral size $L$. In a continuous transition, $T^{*}(L)$ scales as 19] $T^{*}(L)-T^{*} \sim L^{-1 / \nu}\left(1+g L^{-\omega}\right)$, where $g$ is a numerical constant and $\omega$ is an exponent that accounts for corrections to scaling, and is in the range $7 / 4 \leq \omega \leq 2$ for the 2D Ising class [20]. The best multiparameter fit to such scaling form yields $\nu=1.54(47)$, and $\nu=0.94(16)$ on the square and the triangular lattices, together with $\omega=1.6(3)$, and $2.2(2.0)$ respectively, to be compared with $\nu=1$ for the 2D Ising class [21]. Although these results might seem compatible with $2 \mathrm{D}$ Ising universality for the present transition, we believe our numerical evidence favors more strongly a different interpretation. Thus, in marked contrast with 2D Ising and as shown by Fig. 31 the transition in the dLr model is from a phase with finite correlation length to a continuous line of fixed points [in the Renormalization Group (RG) sense], characterized by an infinite value of the correlation length, as occurs in a KT transition [16]. In order to corroborate the latter interpretation, we can try a phenomenological KT-type form for $T^{*}(L)$, namely [19]

$$
T^{*}(L)=T^{*}+\frac{a}{(\log L+b)^{2}},
$$

for constant $a$ and $b$. As seen in Fig. 2 this fit is in very

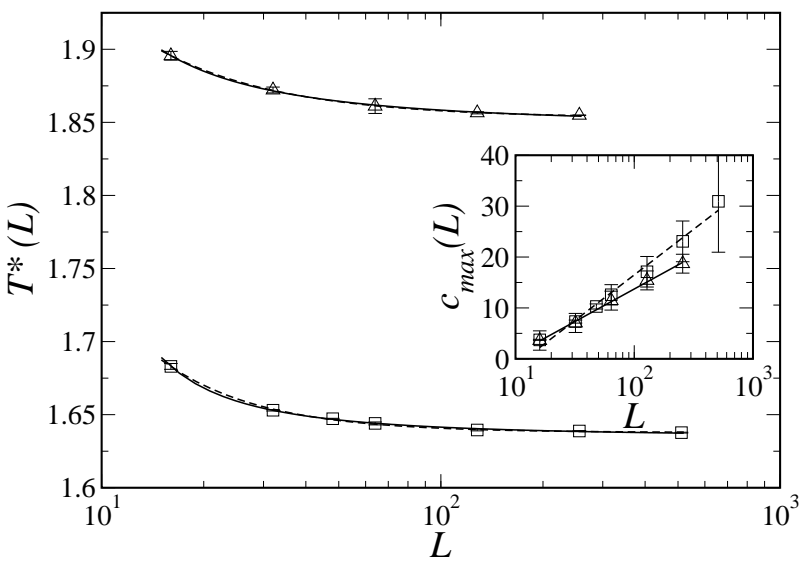

FIG. 4: Transition temperature $T^{*}$ as obtained from Fig. 1 as a function of $L$ for the square $(\square)$ and triangular $(\triangle)$ lattices. For each case, the dashed line is a power-law fit $T^{*}(L)-T^{*} \sim$ $L^{-1 / \nu}$, and the solid line is a fit to Eq. (2). $L=512$ is not employed for the fit due to low statistics. Inset: $c_{\max }(L)$ vs $L$ on the square $(\square)$ and triangular $(\triangle)$ lattices. Lines are logarithmic fits to the data, shown for reference. All bars represent statistical errors and all units are arbitrary.

good agreement with the numerical data for large sizes. We must caution the reader on the well-known feature of the KT transition, that the peak of the specific heat does not occur at the critical temperature but, rather, at a temperature preceding $T_{c}$ 18, 19]. Although the size of this offset can be model-dependent, Fig. 2 indeed provides estimates, $T^{*}=1.63(1)$ on the square lattice, and $T^{*}=1.85(1)$ on the triangular lattice, that are below the corresponding $T_{c}$ values, and are still inside the low $T$ behavior for the spatial correlation functions, see Fig. [3 Thus, the inexistence of an intermediate phase and the fact that the spatial correlations and the specific heat change behavior at different values of $T$ can be hardly reconciled with a single transition of the Ising class.

Our results seem to replace the KTHNY scenario for the dLr model by a single, tensionless, KT-type phase transition. The absence of the hexatic phase may seem surprising when contrasted with the often accepted argument that, for increasing $T$, the surface should first disorder in heights and, then, in slopes. However, this is a sufficient condition for surface roughening, but it is not necessary. For instance, in the $\mathrm{dG}$ model slopes are not disordered at any temperature. It is also conceivable, as is our belief, that heights and slopes disorder at the same temperature in the dLr model. This remarkable result is also against the expectation that discreteness in surface heights renormalizes the surface tension $\sigma$, as it indeed does in the $\mathrm{dG}$ model [16]. For the dLr model, generation of a non-zero $\sigma$ would imply that the asymptotic properties of the high $T$ phase coincide with those of the dG model [1]. In order to explore this possibility, various analytical approaches 15 have been applied to the following continuum analog of the dLr model, introduced 
in the context of growth by MBE [9]:

$$
\frac{\partial h}{\partial t}=-\kappa \nabla^{4} h-\frac{2 \pi V}{a_{\perp}} \sin \left(\frac{2 \pi h}{a_{\perp}}\right)+\sqrt{2 k_{B} T} \zeta
$$

where $\zeta$ is a delta-correlated Gaussian white noise, and $a_{\perp}, V$, are parameters. Although a dynamical RG study for (3) does predict the generation of a non-zero surface tension, numerical simulations of this Langevin equation [9] give results in complete qualitative agreement with those of the dLr model presented here. The discrepancy between the RG arguments and the numerical results for both the discrete and continuum models might be due to inaccuracies in the treatment of model symmetries in the RG studies. Namely, the dLr model can be written as a model for the surface slopes $\mathbf{m} \equiv \nabla_{d} h$, i.e., $\mathcal{H}_{\mathrm{dLr}}=\frac{\kappa}{2} \sum_{\mathbf{r}}\left[\nabla_{d} \cdot \mathbf{m}(\mathbf{r})\right]^{2}$, with the implicit restriction that $\nabla_{d} \times \mathbf{m} \equiv \mathbf{0}$. Thus, the dLr model has larger symmetries than the $\mathrm{dG}$ model, the Hamiltonian being invariant under arbitrary global shifts in the heights, as in the latter, but also in the slopes. Thus the ground state degeneracy here is much larger, minima occurring for all height configurations for which $\nabla_{d} \cdot \mathbf{m}=0$. However, standard perturbative RG analyses [15, 22] are oblivious to such an added complexity in the ground state structure of the model. Perhaps in a related fashion, the zero-vorticity constraint for the slope field may be playing a dynamical rôle in the unbinding of surface defects for $T=T_{c}$ in the
2D melting transition described by the dLr model.

Summarizing, we have found that the dLr model features a single continuous phase transition. Although sizes of our simulations are confined to a regime in which the specific heat still grows logarithmically with $L$, rather than saturating as in proper KT scaling, the combined information from the spatial correlations and the specific heat are consistent with a KT transition. This behavior is remarkably similar to that of the continuum model (3), including the tensionless nature of the high $T$ phase. Progress in the analytical description of these phenomena might improve our understanding of non-perturbative effects in defect-mediated transitions, and of dynamical effects of geometrical constraints (such as the curl-free condition above) in equilibrium systems.

We thank D. R. Nelson and A. Sokal for discussions and A. Sánchez for his collaboration at earlier stages of this work. E. M. thanks DEAS and the Real Colegio Complutense at Harvard for hospitality. Partial financial support from CAM and MEC (Spain) through grants BFM2003-08532-C03-02 and FIS2004-01399 (J. J. R.-L.), BFM2003-07749-C05-01 (R. C.), and BFM2002-04474C02 and FIS2004-01001 (E. M.) is acknowledged. E. M. also acknowledges a Ramón y Cajal contract by MEC. Part of the numerical simulations have been performed on the BIFI PC cluster.
[1] K. J. Strandburg, Rev. Mod. Phys. 60, 161 (1988); J. G. Dash, ibid. 71, 1737 (1999).

[2] A. Pimpinelli and J. Villain, Physics of crystal growth (Cambridge University Press, Cambridge, 1998).

[3] D. Nelson, Defects and Geometry in Statistical Physics (Cambridge university Press, Cambridge, 2002).

[4] K. Bagchi, H. C. Andersen, and W. Swope, Phys. Rev. E 53, 3794 (1996).

[5] C.-F. Chou et al., Science 280, 1424 (1998).

[6] S. T. Chui and J. D. Weeks, Phys. Rev. B 14, 4978 (1976).

[7] D. R. Nelson, Phys. Rev. B 26, 269 (1982).

[8] S. A. Safran, Statistical thermodynamics of surfaces, interfaces, and membranes (Addison-Wesley, Reading, MA, 1994).

[9] E. Moro, R. Cuerno, and A. Sánchez, Phys. Rev. Lett. 78, 4982 (1997)

[10] Here, $\hat{h}(\mathbf{q})$ is the Fourier transform of $h(\mathbf{r})-\bar{h}$, with $\bar{h}$ the mean surface height.

[11] The hexatic behavior $S(q) \sim 1 /\left(\sigma q^{2}\right)$ is also the asymptotics of the free Gaussian model [19], and of the dG model in the high $T$ phase, induced by surface tension.

[12] $C(r)=\left\langle[h(\mathbf{r})-h(\mathbf{0})]^{2}\right\rangle ; C_{d}(s)=\left\langle\left[\nabla_{d} h(\mathbf{s})-\nabla_{d} h(\mathbf{0})\right]^{2}\right\rangle$.

[13] K. J. Strandburg, S. A. Solla, and G. V. Chester, Phys. Rev. B 28, 2717 (1983); D. A. Bruce, Mater. Sci. Forum 4, 51 (1985).

[14] W. Janke and H. Kleinert, Phys. Rev. B 41, 6848 (1990).
[15] E. Moro and R. Cuerno, Phys. Rev. E 63, 036104 (2001); R. Cuerno and E. Moro, ibid. 65, 016110 (2002).

[16] P. Nozières, in Solids far From Equilibrium, edited by C. Godrèche (Cambridge University Press, Cambridge, 1991).

[17] M. E. J. Newman and G. T. Barkema, Monte Carlo Methods in Statistical Physics (Oxford University Press, Oxford, 1999).

[18] J. E. Van Himbergen and S. Chakravarty, Phys. Rev. B 23, 359 (1981); J. E. Van Himbergen, ibid. 25, 5977 (1982).

[19] P. M. Chaikin and T. C. Lubensky, Principles of condensed matter physics (Cambridge University Press, Cambridge, 1995).

[20] J. Salas and A. Sokal, J. Stat. Phys. 98 (2000) 551-588.

[21] A simpler minded fit to a standard scaling form (see Fig. 2] provides $\nu=0.71(4)$ and $\nu=0.81(4)$ on the square and the triangular lattices, respectively. Besides the apparent lack of universality, these values of $\nu$, along with our result that the specific heat exponent $\alpha \leq 0$, would actually imply violation of the hyperscaling relation [19] $\alpha=2-\nu d$, for $d=2$.

[22] Y. Levin and K. A. Dawson, Phys. Rev. A 42, 3507 (1990); B. Kahng and K. Park, Int. J. Mod. Phys. B 10, 543 (1996). 